\title{
Knockdown of long noncoding RNA colorectal neoplasia differentially expressed inhibits hepatocellular carcinoma progression by mediating the expression of nuclear autoantigenic sperm protein
}

\author{
YUNGANG ZHU $^{1 *}$, BAOGUO LI ${ }^{2 *}$, GUOPING XU ${ }^{3}, \mathrm{CHANGRUI} \mathrm{HAN}^{1}$ and GANG XING ${ }^{1}$ \\ ${ }^{1}$ Radiology Department, Tianjin Teda Hospital, Tianjin 300457; ${ }^{2}$ Department of Interventional Treatment, \\ National Clinical Research Center for Cancer, Key Laboratory of Cancer Prevention and Therapy, \\ Tianjin's Clinical Research Center for Cancer, Tianjin Medical University Cancer Institute and Hospital, Tianjin 300060; \\ ${ }^{3}$ Medical Imaging Department, The Second Hospital of Tianjin Medical University, Tianjin 300211, P.R. China
}

Received April 16, 2021; Accepted August 4, 2021

DOI: $10.3892 /$ or.2021.8203

\begin{abstract}
Long noncoding RNAs (lncRNAs) play critical roles in the tumorigenesis and progression of hepatocellular carcinoma (HCC). As the most common malignant cancer type in humans, $\mathrm{HCC}$ poses a great threat to human health. However, the function of lncRNA colorectal neoplasia differentially expressed (CRNDE) in HCC has not been extensively studied. The chief aim of the present study was to reveal the potential role of CRNDE in HCC. Expression levels of CRNDE in HCC tissues and cell lines were detected by reverse transcription-quantitative (RT-q) PCR, and Cell Counting kit 8, wound-healing and Transwell assays were used to evaluate the influences of CRNDE on in vitro cellular proliferation, migration and invasiveness, respectively. The interaction between CRNDE and microRNA (miR)-29c-3p was determined by dual-luciferase reporter assay, and rescue experiments were conducted to evaluate the interactive relationships between CRNDE and miR-29c-3p or nuclear autoantigenic sperm protein (NASP). CRNDE was found to be upregulated in HCC tissues and cells, and to be positively associated with the poor prognosis of patients with HCC. Furthermore, CRNDE-knockdown suppressed cell proliferation, migration and invasion abilities. Bioinformatics and RT-qPCR analysis indicated miR-29c-3p as a potential target of
\end{abstract}

Correspondence to: Professor Gang Xing, Radiology Department, Tianjin Teda Hospital, 65 Third Avenue, Binhai New Area, Tianjin 300457, P.R. China

E-mail: doctorxing2021@126.com

*Contributed equally

Key words: long noncoding RNA colorectal neoplasia differentially expressed, hepatocellular carcinoma, microRNA-29c-3p, nuclear autoantigenic sperm protein, oncogenic
CRNDE. In line with previous reports, as a tumor suppressor, downregulated expression of miR-29c-3p was observed in HCC. In addition, the present study revealed that miR-29c-3p directly targeted NASP. NASP expression was markedly elevated following transfection with an miR-29c-3p inhibitor, while knocking down CRNDE inhibited NASP expression. Moreover, the effects of CRNDE and NASP on HCC cells were reversed by miR-29c-3p. Collectively, the results of the present study revealed that CRNDE was upregulated and exerted an oncogenic role in HCC by targeting miR-29c-3p, and that the upregulation of CRNDE also promoted NASP expression. These findings indicate a novel role for CRNDE in the progression of $\mathrm{HCC}$.

\section{Introduction}

In the past few decades, hepatocellular carcinoma (HCC) has become one of the most common human malignancies, accounting for $>70 \%$ of all liver cancer cases worldwide (1), and was the third leading cause of cancer-related death worldwide in 2017 (2). Although considerable advances have been made in HCC diagnosis and treatment, the prognosis of patients is still unsatisfactory (3). The 5-year survival rate of HCC remains at a low level, which may be due to the high recurrence rate after surgical resection (4). Studies have indicated that the primary cause of HCC is infection with hepatitis $\mathrm{B}$ and $\mathrm{C}$ virus. However, diabetes and excessive drinking can also contribute to HCC (5). Therefore, a more thorough exploration of $\mathrm{HCC}$ pathogenesis is imperative to developing new diagnostic methods and identifying therapeutic targets.

Long noncoding RNAs (lncRNAs), >200 nucleotides in length, have no protein-coding function (6). As a type of noncoding RNA, the functions of lncRNAs have been widely investigated during the past decade. Increasing numbers of studies have reported that dysregulation of lncRNA expression is closely associated with the occurrence and development of cancers (7-11). Research has also indicated the critical roles of IncRNAs in hepatocarcinogenesis, with regard to cellular 
proliferation, apoptosis and metastasis (12). For example, expression of LINRIS was upregulated in CRC tissues and is associated with poor overall survival, and LINRIS stabilizes insulin-like growth factor $2 \mathrm{mRNA}$-binding protein 2 to promote colorectal cancer progression (13). Furthermore, lncRNA anti-differentiation ncRNA was found to regulate the invasion and metastasis abilities of breast cancer cells by affecting the stability of histone-lysine N-methyltransferase EZH2 (14). Expression levels of lncRNA colorectal neoplasia differentially expressed (CRNDE) have been found to increase in various types of cancer. For example, higher expression of lncRNA CRNDE promoted cellular proliferation and chemoresistance by targeting microRNA (miR)-181a-5p-Wnt/beta-catenin signaling in colorectal cancer (15). CRNDE also bound and reduced the protein stability of serine/arginine-rich splicing factor 6 to regulate the chemoresistance of gastric cancer (16). In the present study, the molecular mechanism of CRNDE in $\mathrm{HCC}$ was further explored.

MicroRNAs (miRNAs/miRs) are also involved in the progression of HCC. For example, miR-29c-3p expression was downregulated in colorectal cancer tissues, and inhibited cancer progression by negatively regulating circ-001971 and VEGFA (17). Low expression levels of miR-29c-3p were associated with shorter overall survival times of patients with HCC, while overexpression of miR-29c-3p markedly inhibited cellular proliferation and tumor growth (18). Nuclear autoantigenic sperm protein (NASP) is reported to be upregulated in gastric cancer, ovarian cancer and hepatocellular carcinoma (19-21). However, the function of the miR-29c-3p-NASP axis in HCC remains unclear.

In the present study, the expression level of CRNDE in HCC was assessed using normal non-tumor tissue data from the Gene Expression Profiling Interactive Analysis (GEPIA) database and reverse transcription-quantitative (RT-q) PCR. miR-29c-3p was predicted as a potential target of CRNDE, and its CRNDE regulatory role was investigated. In addition, the effects of the CRNDE/miR-29c-3p/NASP axis on cellular proliferation, migration and invasiveness, and the underlying mechanisms of CRNDE/miR-29c-3p/NASP in HCC, were determined.

\section{Materials and methods}

Clinical specimens. A total of 40 pairs of HCC and adjacent-normal tissues were collected from The Second Hospital of Tianjin Medical University (Tianjin, China) between January 2017 and December 2019. HCC diagnosis was primarily confirmed by tumor marker detection and liver imaging examinations. Patients were aged $<50$ years old $(n=17)$ and $\geq 50$ years old $(n=23), 22$ of which were male, and 18 female. Patient inclusion criteria included: i) The patient was diagnosed with primary liver cancer; and ii) the patient understood the purpose of the study and provided written informed consent. Exclusion criteria included: i) Patients diagnosed with other tumors or liver insufficiency; ii) patients experiencing mental illness; and iii) patients with other major diseases other than the tumor. The distance between the tumor tissue and healthy tissues was $\geq 3 \mathrm{~cm}$, and tissues were immediately frozen in the liquid nitrogen following resection. The patient clinicopathological features are presented in Table I.
Surgical indications include the tumor lesion not exceeding half of the liver, no distant metastases, and that patients exhibited normal liver function. None of the enrolled patients had received any pre-surgical treatment. The study was approved by the Ethical Committee of the Second Hospital of Tianjin Medical University (approval no. KY2020K093), and written informed consent was obtained from each patient.

Cell culture. Normal liver THLE-2 cells and liver cancer cells (SNU-398 and Huh7) were purchased from the American Type Culture Collection. All cell lines were authenticated by STR profiling. The cells were cultured in RPMI 1640 medium supplemented with $10 \%$ fetal bovine serum (FBS; both Gibco; Thermo Fisher Scientific, Inc.), and maintained at $37^{\circ} \mathrm{C}$ in an incubator containing $5 \% \mathrm{CO}_{2}$.

Transfection. Lentivector-mediated short hairpin (sh) RNA CRNDE (shCRNDE-1, 5'-GGTTAAGCTGTATTTGAT TGC-3'; shCRNDE-2, 5'-GATGTGTTTCAATCTAGA TGC-3'; and shCRNDE-3, 5'-GCTCGAGTGGTTTAA ATATGT-3') and the negative control plasmids (shNC; 5'-CAACAAGATGAAGAGCACCAA-3') were synthesized by Guangzhou RiboBio Co., Ltd. The CRNDE overexpression plasmid and the empty negative control plasmid (pcDNA3.1) were purchased from Shanghai GenePharma Co., Ltd. Briefly, CRNDE-encoding cDNA was amplified and cloned into the pcDNA3.1 plasmid; sequence sources for CRNDE and NASP are Accession NR_034105.4 and NM_001195193, respectively. The negative control (NC) mimics, miR-29c-3p mimics, miR-29c-3p inhibitor and NC inhibitor were purchased from Guangzhou RiboBio Co., Ltd., according to the mature miR-29c-3p sequence (5'-UAG CACCAUUUGAAAUCGGUUA-3'). At 70\% confluence, HCC cells were transfected using Lipofectamine ${ }^{\circledR} 3000$ (Invitrogen; Thermo Fisher Scientific, Inc.) according to the manufacturer's instructions. Briefly, $1 \mu \mathrm{g}$ nucleic acid was used to transfect cells; the nucleic acid and Lipofectamine were separately diluted using Opti-MEM ${ }^{\circledR}$ (Invitrogen; Thermo Fisher Scientific, Inc.), and then mixed and incubated at room temperature for $5 \mathrm{~min}$. Then, $48 \mathrm{~h}$ after transfection at $37^{\circ} \mathrm{C}$, the cells were harvested for subsequent experimentation.

$R N A$ extraction and $R T-q P C R$. Total RNA was extracted from tissues and cells using TRIzol ${ }^{\circledR}$ reagent (Invitrogen; Thermo Fisher Scientific, Inc.) per the manufacturer's instructions, and quantified with a NanoDrop 2000 instrument (Thermo Fisher Scientific, Inc.). Then, $1 \mu \mathrm{g}$ RNA was reverse transcribed to cDNAs using the Reverse Transcription System kit (Invitrogen; Thermo Fisher Scientific, Inc.) in a $20 \mu 1$ reaction. qPCR was then performed using the $\mathrm{SYBR}^{\circledR}$ Premix EX Taq ${ }^{\mathrm{TM}}$ kit (Takara Bio, Inc.) with a Real-Time PCR System (Thermo Fisher Scientific, Inc.): Total reaction (20 $\mu \mathrm{l}$ ) consisted of $10 \mu \mathrm{l}$ master mix (TaqMan), $1 \mu \mathrm{l}$ forward and reverse primers, $2 \mu \mathrm{l}$ cDNA and $7 \mu \mathrm{l}$ DEPC water. Reaction conditions: i) $95^{\circ} \mathrm{C}$ for $5 \mathrm{~min}$; and ii) 40 cycles of $95^{\circ} \mathrm{C}$ for $10 \mathrm{sec}, 60^{\circ} \mathrm{C}$ for $30 \mathrm{sec}$, and $72^{\circ} \mathrm{C}$ for $30 \mathrm{sec}$. Gene expression was quantified using the $2^{-\Delta \Delta \mathrm{Cq}}$ method (22). The expression of CRNDE and NASP was normalized to that of GAPDH (the internal control), and miR-29c-3p 
Table I. Association between CRNDE expression and the clinicopathological characteristics of patients with HCC ( $\mathrm{n}=40$ ).

\begin{tabular}{|c|c|c|c|c|}
\hline \multirow[b]{2}{*}{ Characteristics } & \multirow[b]{2}{*}{$\mathrm{n}=40$} & \multicolumn{2}{|c|}{ CRNDE expression } & \multirow[b]{2}{*}{ P-value } \\
\hline & & Low $(n=20)$ & High $(n=20)$ & \\
\hline \multicolumn{5}{|l|}{ Age, years } \\
\hline$<50$ & 17 & 8 & 9 & \\
\hline$\geq 50$ & 23 & 12 & 11 & 0.749 \\
\hline \multicolumn{5}{|l|}{ Sex } \\
\hline Male & 22 & 14 & 8 & \\
\hline Female & 18 & 6 & 12 & 0.057 \\
\hline \multicolumn{5}{|l|}{ Tumor size, $\mathrm{cm}$} \\
\hline$\leq 5$ & 14 & 10 & 4 & \\
\hline$>5$ & 26 & 10 & 16 & 0.047 \\
\hline \multicolumn{5}{|l|}{ TNM stage } \\
\hline $\mathrm{I}+\mathrm{II}$ & 18 & 13 & 5 & \\
\hline III+IV & 22 & 7 & 15 & 0.011 \\
\hline
\end{tabular}

CRNDE, lncRNA colorectal neoplasia differentially expressed.

expression was normalized to U6. qPCR was performed with the following primers: CRNDE forward, 5'-AAATTC ATCCCAAGGCTGGT-3' and reverse, 5'-AAACCACTC GAGCACTTTGA-3'; NASP forward, 5'-AGATTGGGA ACCTAGAGCTTGC-3' and reverse, 5'-ACTTCTCCGAGT TTAAGATGTGC-3'; miR-29c-3p forward, 5'-ACACTC CAGCTGGTAGCACCATTGAAAT-3' and reverse, 5'-TGG TGTCGTGGAGTCG-3'; miR-29a-3p forward, 5'-GGGTAG CACCATCTGAAAT-3' and reverse, 5'-CAGTGCGTGTCG TGGAGT-3'; miR-146a-5p forward, 5'-GGCGATGAGAAC TGAATTCCA-3' and reverse, 5'-GCAGGGACCGTGGT-3'; miR-455-3p forward, 5'-GCCGGATCCAGTCGTGTT GCTTTATCTACAGAGC-3' and reverse, 5'-GCCGAATTC TCAGAGCGGTCAAGCAGTGA-3'; miR-28-5p forward, 5'-TGGTGTCGTGGGTCGA-3' and reverse, 5'-CTCGCT TCGGCAGCACA-3'; miR-338-3p forward, 5'-GGGTCC AGCATCAGTGATT-3' and reverse, 5'-GTGCAGGGT CCGAGGT-3'; GAPDH forward, 5'-AATGGACAACTG GTCGTGGAC-3' and reverse, 5'-CCCTCCAGGGGA TCTGTTTG-3'; and U6 forward, 5'-AGTAAGCCCTTG CTGTCAGTG-3' and reverse, 5'-CCTGGGTCTGATAAT GCTGGG.

Cell viability assay. The Cell Counting kit 8 (CCK-8; Dojindo Molecular Technologies, Inc.) was used to evaluate cellular proliferation after transfection. A total of $1 \times 10^{3}$ transfected cells per well were seeded into 96-well plates. Then, $10 \mu \mathrm{l} \mathrm{CCK-8}$ solution was added to each well at the indicated transfection time points $(24,48$ and $72 \mathrm{~h}$ ) and incubated for a further $2 \mathrm{~h}$ according to the manufacturer's instructions. The absorbance was detected at $450 \mathrm{~nm}$ using a micro-plate reader. Cell viability was calculated according to the manufacturer's instructions.

Wound-healing assay. Transfected cells were seeded into a 6 -well plate. When reaching confluence, a wound was created in the monolayer using a $10-\mu 1$ pipette tip and the cells were cultured in medium without serum. Images of wound width were captured at 0 and $24 \mathrm{~h}$ using an inverted microscope (Thermo Fisher Scientific, Inc.). The width of each wound was measured and the fold change was calculated.

Dual-luciferase reporter assay. To confirm the interaction between miR-29c-3p and CRNDE, as well as miR-29c-3p and NASP, wild-type (WT) and mutant-type (MUT) sequences of CRNDE and NASP (containing the putative binding site of miR-29c-3p) were cloned into the pGL3 reporter vector (Promega Corporation). 293T cells were co-transfected with the WT or MUT reporter plasmids, and miR-29c-3p mimics or NC mimics using Lipofectamine ${ }^{\circledR} 3000$ (Invitrogen; Thermo Fisher Scientific, Inc.). After 48 h, the cells were collected and lysed. The fluorescence intensity was measured using the Dual-Luciferase Reporter Assay System (Promega Corporation) and normalized by comparison with Renilla luciferase activity, according to the manufacturer's instructions.

Transwell assay. To assess cellular migration and invasion ability, $1 \times 10^{5}$ cells were seeded into the upper chamber of Transwell inserts with serum-free medium to assess migration, and medium containing $10 \%$ FBS was added to the lower chambers. For the invasion assay, the upper chamber was pre-coated with Matrigel (BD Biosciences, 1:8 dilution) at $37^{\circ} \mathrm{C}$ for $7 \mathrm{~h}$. Cells were maintained in an incubator at $37^{\circ} \mathrm{C}$ prior to fixing. After $24 \mathrm{~h}$, the cells were fixed with $4 \%$ paraformaldehyde for $10 \mathrm{~min}$ and stained with $0.4 \%$ crystal violet for $10 \mathrm{~min}$ at room temperature. Then, the stained cells were washed with PBS and observed using an inverted microscope (Thermo Fisher Scientific, Inc.). The number of cells was calculated in five randomly selected visual fields using ImageJ 1.51 software (National Institutes of Health).

Western blotting. Total protein samples were extracted form cells using RIPA lysis buffer (Beyotime Institute of 
Biotechnology) with PMSF. Protein concentration was quantified using a BCA kit (Beyotime Institute of Biotechnology). Equal amounts of protein $(30 \mu \mathrm{g})$ were separated by $10 \%$ SDS-PAGE and transferred to PVDF membranes. The Membranes were then blocked with 5\% non-fat milk for $1 \mathrm{~h}$ at room temperature, and incubated with the primary antibodies overnight at $4^{\circ} \mathrm{C}$. Then, the membranes were incubated with the corresponding HRP-conjugated secondary antibodies for $1 \mathrm{~h}$ at room temperature. The protein bands were visualized using an ECL kit (Beyotime Institute of Biotechnology). The gray values of the protein bands were quantified using ImageJ 1.51 software (National Institutes of Health) and relative expression levels were calculated. The following antibodies were employed: Anti-NASP (1:1,000; cat. no. ab181169; Abcam), anti-N cadherin (1:3,000; cat. no. ab76011; Abcam), anti-E cadherin (1:1,000; cat. no. ab231303; Abcam), anti-vimentin antibody (1:1,000; cat. no. ab16700; Abcam), anti-GAPDH (1:5,000; cat. no. ab8245; Abcam), goat anti-rabbit (1:3,000; cat. no. 7074; CST Biological Reagents Co., Ltd.) and horse anti-mouse (1:3,000; cat. no. 7076; CST Biological Reagents Co., Ltd.).

Immunohistochemistry. Tissues were fixed with $4 \%$ paraformaldehyde at room temperature for $\geq 48 \mathrm{~h}$. Paraffin sections of $5 \mu \mathrm{m}$ were cut from the tumor tissues of patients with HCC, and were dewaxed with xylene for $3 \mathrm{~h}$ at room temperature, and rehydration in a descending alcohol series (absolute ethanol, $10 \mathrm{~min}$; methanol with 3\% hydrogen peroxide, $10 \mathrm{~min}$; $90 \%$ alcohol, $10 \mathrm{~min}$; $70 \%$ alcohol, $5 \mathrm{~min}$ ). The samples were then washed with $\mathrm{dd}_{2} \mathrm{O}$. Antigen retrieval was conducted at $95^{\circ} \mathrm{C}$ for $15 \mathrm{~min}$, then the samples were cooled to room temperature andwashed with $\mathrm{ddH}_{2} \mathrm{O}$ once again. The sections were blocked with $10 \%$ goat serum at room temperature for $1 \mathrm{~h}$, and then incubated with anti-NASP (1:100; cat. no. sc-514669; Santa Cruz Biotechnology, Inc.) at $4^{\circ} \mathrm{C}$ overnight. After incubation with an anti-mouse HRP-conjugated secondary antibody $(1: 1,000$; cat. no. 7076; CST Biological Reagents Co., Ltd.), the sections were stained with DAB at room temperature for $10 \mathrm{~min}$, and hematoxylin at room temperature for $5 \mathrm{~min}$, and NASP expression was observed using a light microscope.

Bioinformatics analysis. CRNDE expression data were obtained from The Cancer Genome Atlas (TCGA) in GEPIA (http://gepia.cancer-pku.cn/) and the Lnc2Cancer 3.0 (http://bio-bigdata.hrbmu.edu.cn/lnc2cancer/index.html) database. Differentially expressed miRNAs were screened in dbDEMC 2.0 (https://www.picb.ac.cn/dbDEMC/index.html). Target genes were analyzed using UALCAN (http://ualcan.path. uab.edu/). Potential target miRNAs of CRNDE were predicted by starBase (http://starbase.sysu.edu.cn). Target genes of miRNA were predicted by starBase, miRWalk (http://mirwalk. umm.uni-heidelberg.de/), TargetScan (http://www.targetscan. org/vert_71/), miRDB (http://mirdb.org/) and ONCOMIR (http://www.oncomir.org/oncomir/index.html).

Statistical analysis. Results are presented as the mean $\pm \mathrm{SD}$, and were analyzed using SPSS 19.0 (IBM Corp) and GraphPad Prism 8.0 Software (GraphPad Software, Inc.). One-way ANOVA and Tukey's test were used for multiple comparison analyses. All data from normal vs. tumor tissues were analyzed using paired t-tests. All other comparisons between two groups were analyzed by unpaired t-tests. Associations between CRNDE expression and clinicopathological characteristics were assessed using the $\chi^{2}$ test. All experiments were carried out in triplicate, and $\mathrm{P}<0.05$ was considered to indicate a statistically significant difference.

\section{Results}

CRNDE expression is elevated in HCC tissues. First, CRNDE expression was detected in HCC tissues using online database analysis and RT-qPCR. Results from TCGA revealed that the expression of CRNDE was significantly increased in HCC tissues compared with adjacent-normal tissues (Fig. 1A), and a similar result was observed in the Lnc2Cancer 3.0 database (Fig. 1B). CRNDE expression was also increased in a number of other cancer types, aside from HCC (Fig. 1C). Furthermore, RT-qPCR was performed to assess the expression of CRNDE in HCC and non-cancer tissues. The results indicated that CRNDE expression was significantly elevated in HCC tissues (Fig. 1D). Thus, these results suggested that $\mathrm{CRNDE}$ is positively associated with HCC progression.

CRNDE-knockdown suppresses HCC cell proliferation, migration and invasiveness. Next, the expression levels of CRNDE were assessed in normal liver and HCC cell lines. Concertedly, CRNDE expression was upregulated in cancer cells, but not in normal liver cells (Fig. 2A). To determine its function in HCC cells, CRNDE expression was silenced in SNU-398 and Huh7 cells via shRNA-CRNDE transfection, and confirmed by RT-qPCR. Due to superior knockdown efficiency, shCRNDE-2 and shCRNDE-3 were selected for further experimentation (Fig. 2B). Subsequently, a CCK-8 assay was used to evaluate the proliferative capacity of cells transfected with shCRNDE, which was found to be significantly inhibited by CRNDE-knockdown (Fig. 2C). Moreover, Transwell and wound-healing assays revealed that the migratory and invasive capacities were suppressed when CRNDE was knocked down (Fig. 2D-F). These results demonstrated that CRNDE-knockdown inhibited the proliferation and metastatic potential of HCC cells in vitro.

$C R N D E$ targets and negatively regulates $\mathrm{miR}-29 \mathrm{c}-3 p$ expression. Increasing reports have indicated that lncRNAs can act as competing endogenous RNAs (ceRNAs) to miRNAs $(23,24)$. In the present study, 60 potential targets of CRNDE were predicted using the starBase database. Furthermore, differentially expressed miRNAs were screened in association with multiple cancers via bioinformatics analysis (Fig. 3A). In HCC, six miRNAs with low expression were selected, and their expression assessed in SNU-398 and Huh7 cells. The RT-qPCR results showed that among the six miRNAs, miR-29c-3p expression was the lowest expressed in HCC cells (Fig. 3B). Therefore, miR-29c-3p was selected as a potential target miRNA of CRNDE. The WT and MUT binding sequence between CRNDE and miR-29c-3p is presented in Fig. 3C. To verify the interaction between CRNDE and miR-29c-3p, a dual-luciferase reporter assay was performed, revealing that 


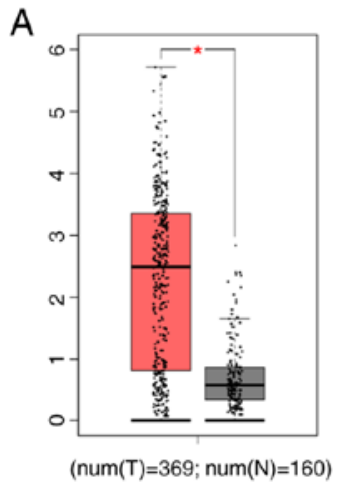

\section{$\mathrm{C}_{8}$}

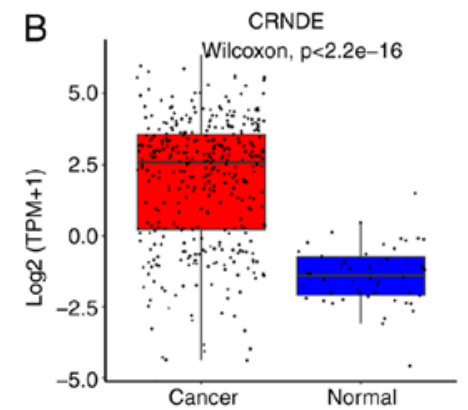

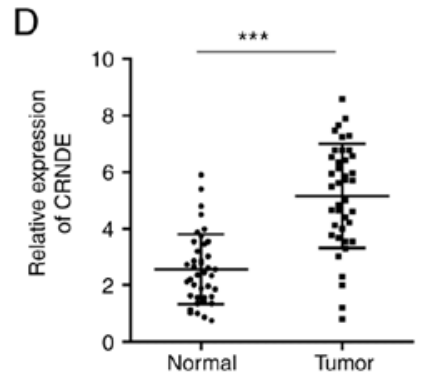

Figure 1. CRNDE is highly expressed in HCC tissues and cells. (A) CRNDE expression analysis in HCC and non-tumor tissues; data were obtained from The Cancer Genome Atlas in the Gene Expression Profiling Interactive Analysis database. (B) Lnc2Cancer 3.0 database was used to analyze CRNDE expression in HCC. (C) CRNDE expression in different types of cancer. (D) Reverse transcription-quantitative PCR was used to determine CRNDE expression levels in HCC and adjacent-normal tissues. ${ }^{*} \mathrm{P}<0.05$ and ${ }^{* * *} \mathrm{P}<0.001$. CRNDE, IncRNA colorectal neoplasia differentially expressed; HCC, hepatocellular carcinoma; T, tumor; N, normal.

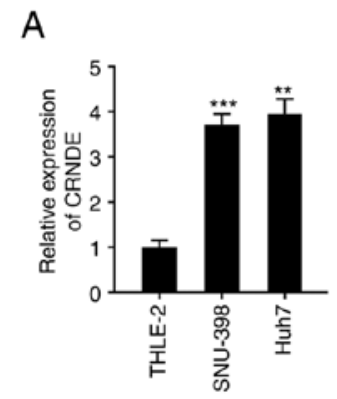

$\mathrm{D}$

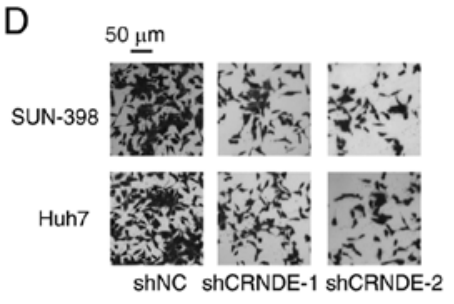

$\mathrm{F}$

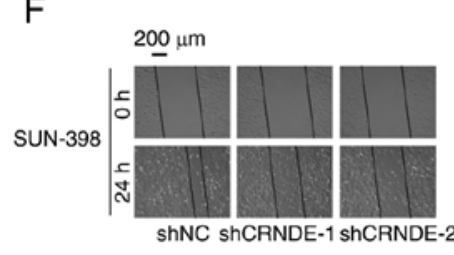

B

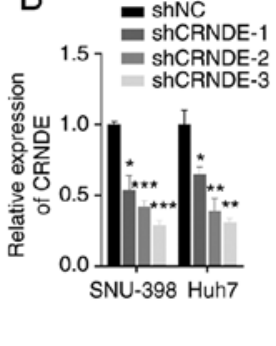

C

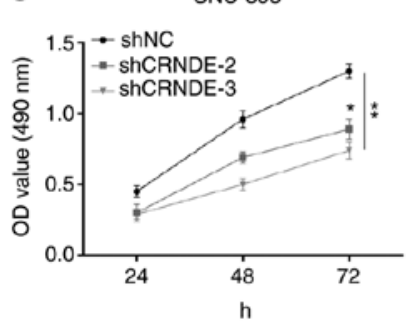

Huh7

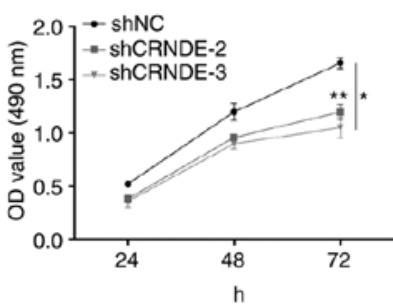

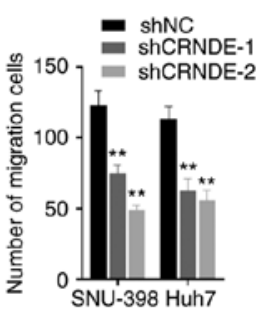
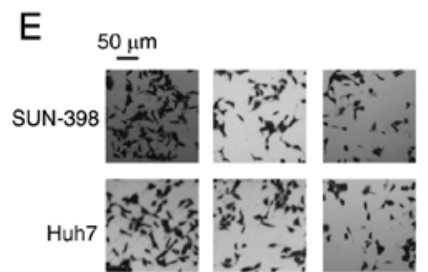

ShNC ShCRNDE-1 ShCRNDE-2
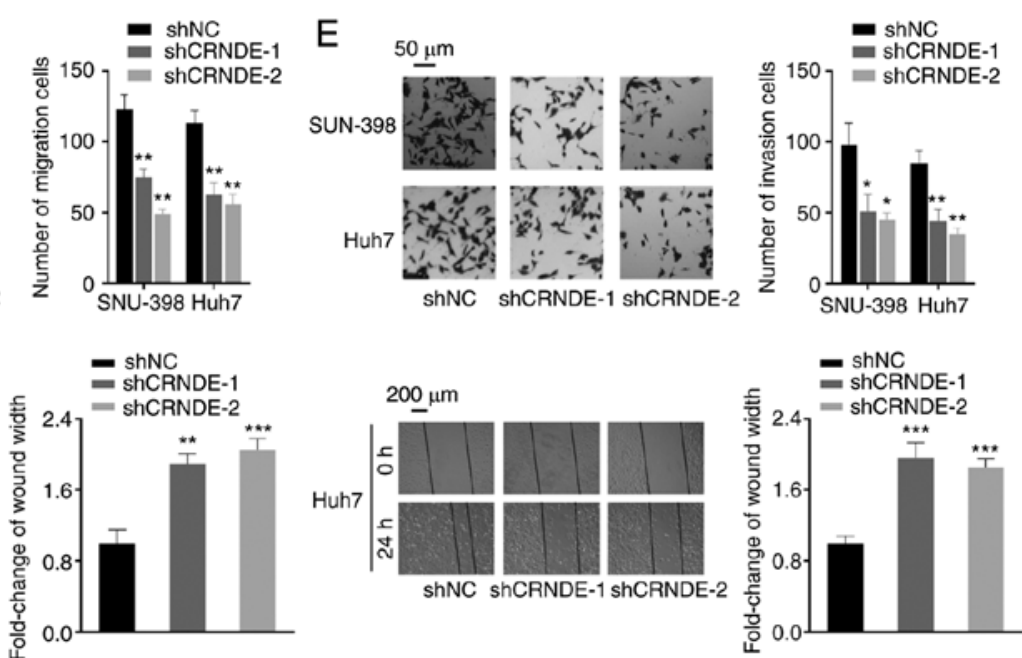

Figure 2. CRNDE-knockdown inhibits HCC cell proliferation, migration and invasiveness. (A) Relative expression levels of CRNDE in normal liver cells and liver cancer cells. (B) SNU-398 and Huh7 cells were transfected with shNC, shCRNDE-1, shCRNDE-2 or shCRNDE-3, and CRNDE expression was determined by reverse transcription-quantitative PCR. (C) HCC cell proliferation was determined by Cell Counting kit 8 analysis after shCRNDE transfection. (D and E) CRNDE-knockdown inhibited the migration and invasiveness of HCC cells. (F) Migration ability was detected by wound-healing assays in SNU-398 and Huh7 cells transfected with shNC, shCRNDE-1 or shCRNDE-2. ${ }^{*} \mathrm{P}<0.05,{ }^{* * *} \mathrm{P}<0.01$ and ${ }^{* * * *} \mathrm{P}<0.001$ vs. THLE-2 or shNC. CRNDE, lncRNA colorectal neoplasia differentially expressed; HCC, hepatocellular carcinoma; sh, short hairpin (RNA); NC, negative control. 
A
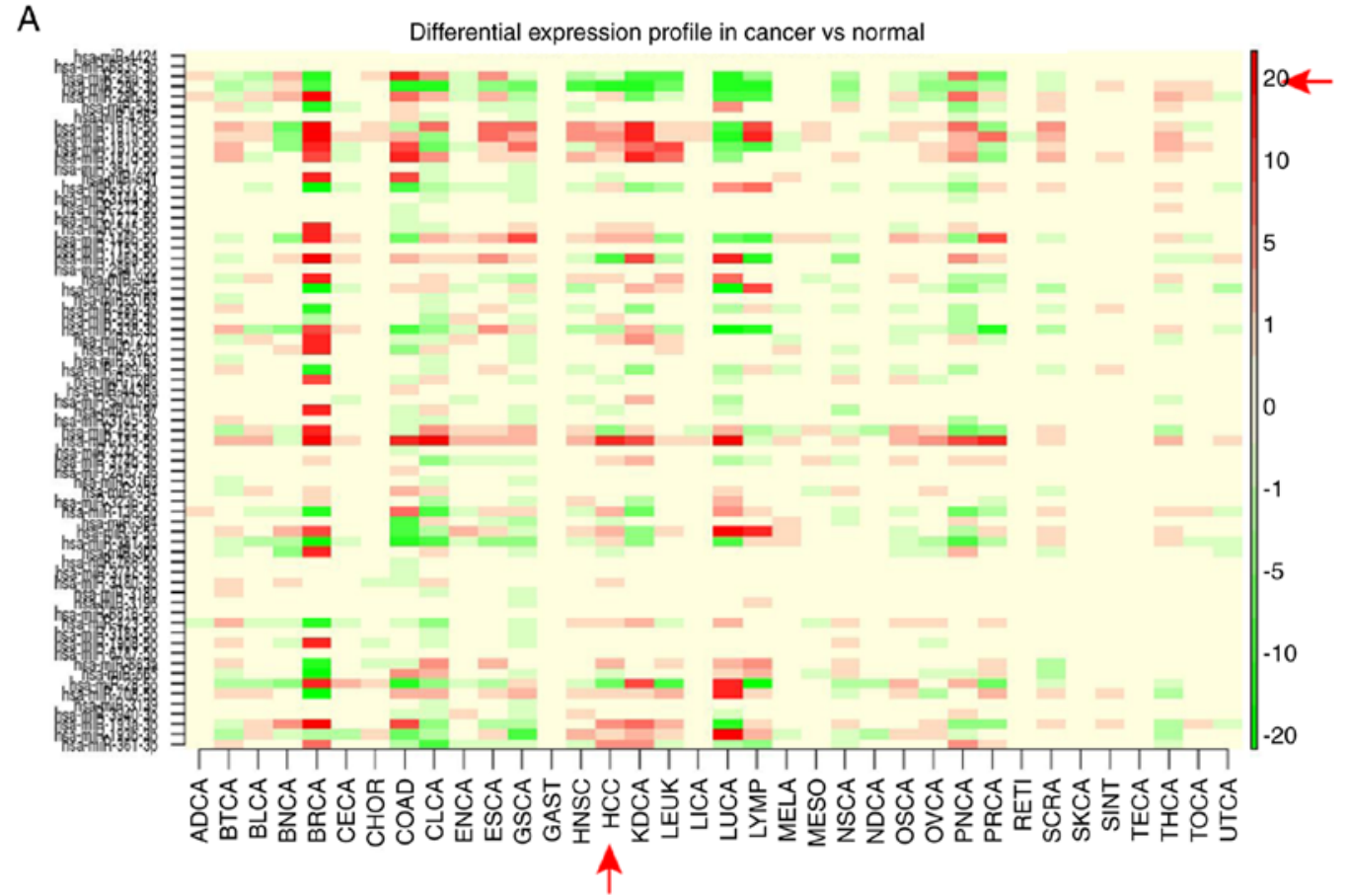

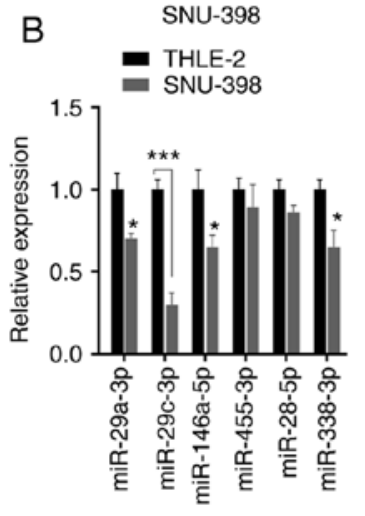

$\mathrm{D}$

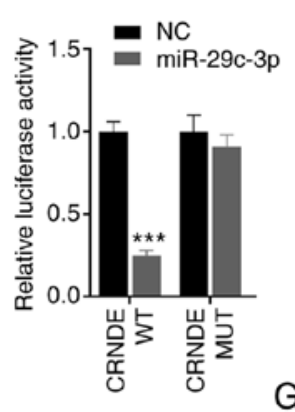

Huh7

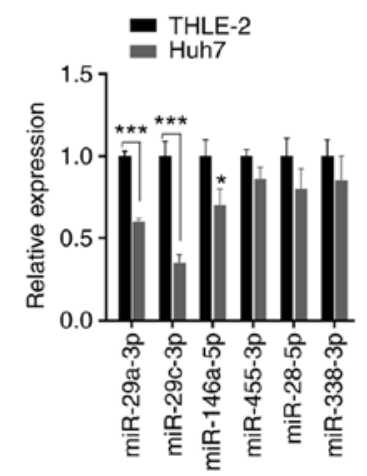

$\mathrm{E}$

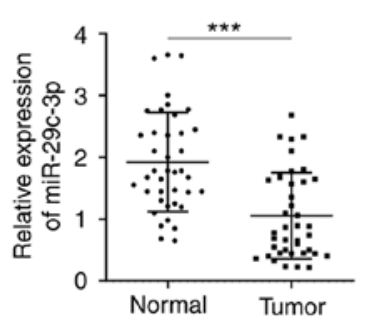

$\mathrm{F}$

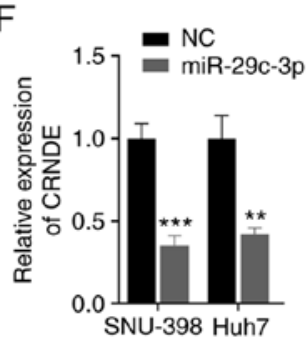

CRNDE-WT 5' gcuaUGCUUAUUCGUGGUGCUU $3^{\prime}$

miR-29c-3p: $3^{\prime}$ auugGCUAAAGUUUACCACGAu 5'

CRNDE-MUT $5^{\prime}$ gCuaUGCUUAUUCGAUCACGAU $3^{\prime}$

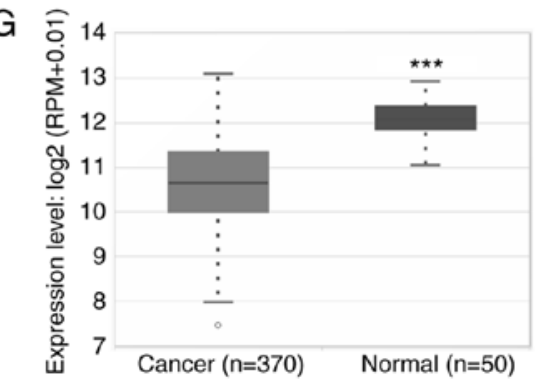

Figure 3. miR-29c-3p is a target of CRNDE. (A) Heat map of miRNA expression in various cancer types. (B) Expression levels of six target miRNAs of CRNDE were determined in SNU-398 and Huh7 by RT-qPCR. (C) Binding sites between CRNDE and miR-29c-3p were predicted using the starBase database. (D) Interaction between CRNDE and miR-29c-3p was confirmed by dual-luciferase reporter assay. (E) miR-29c-3p expression was quantified in HCC and adjacent-normal tissues by RT-qPCR. (F) CRNDE expression in HCC cells transfected with NC mimics or miR-29c-3p mimics. (G) StarBase database revealed decreased expression of miR-29c-3p in HCC tissues compared with adjacent-normal tissues. ${ }^{*} \mathrm{P}<0.05,{ }^{* * *} \mathrm{P}<0.01$ and ${ }^{* * * *} \mathrm{P}<0.001$. miR, microRNA; CRNDE, IncRNA colorectal neoplasia differentially expressed; HCC, hepatocellular carcinoma; RT-q, reverse transcription-quantitative; WT, wild-type; MUT, mutant-type. 


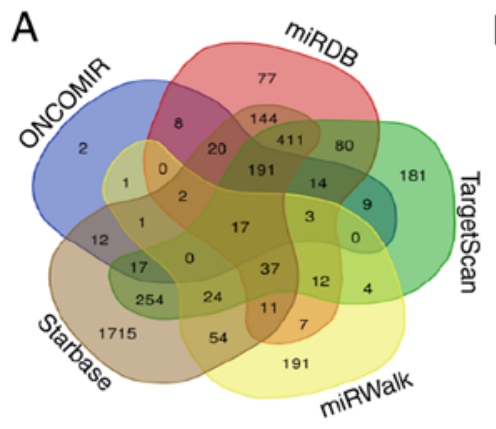

B

C

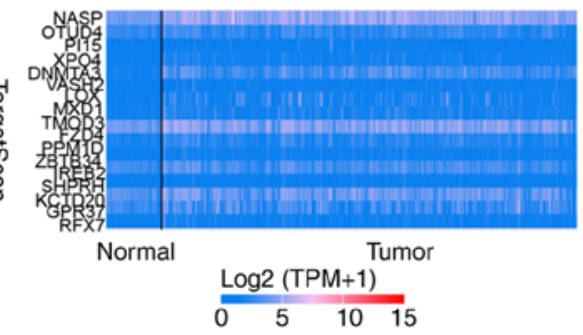

NASP-WT $5^{\prime}$...GCCUUUGUUUUCCAAUGGUGCUA...3'

miR-29c-3p : $3^{\prime}$ AUUGGCUAAAGUCUACCACGAU 5'

NASP-MUT $5^{\prime}$...GCCUUUGUUUUCCAAUGGUGCUA....3'

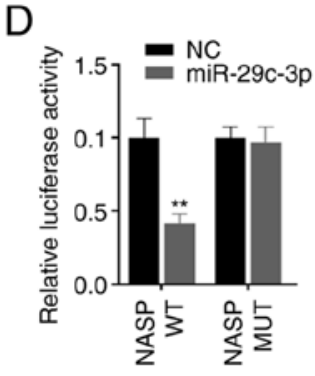

E

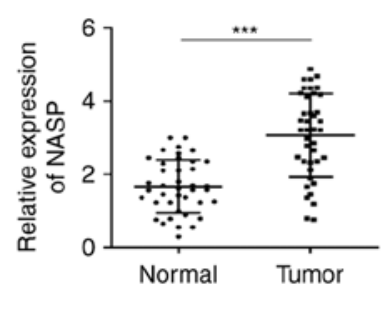

G

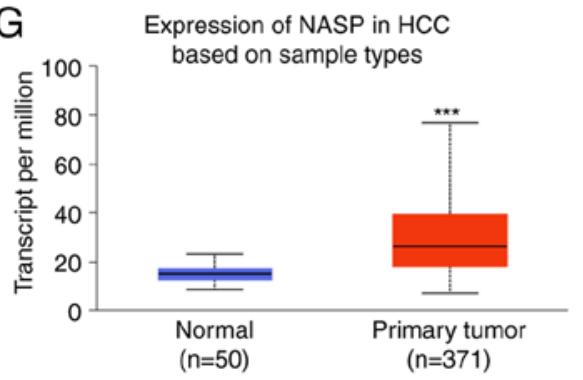

$\mathrm{H}$

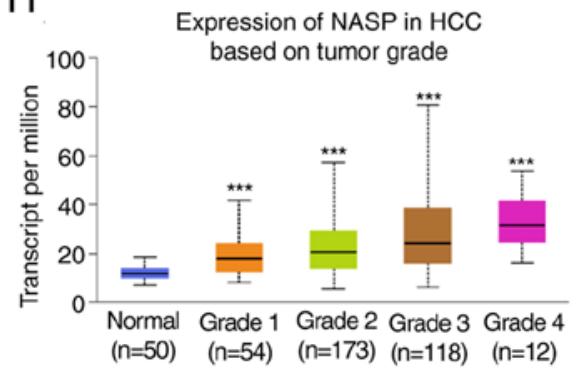

I

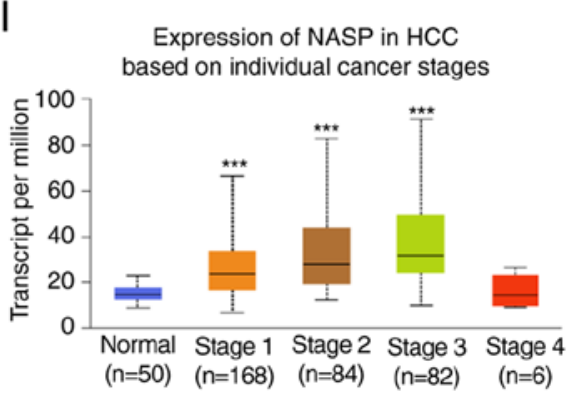

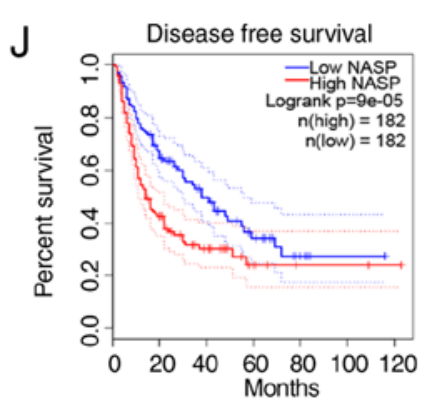

Figure 4. miR-29c-3p targets NASP and NASP expression is increased in patients with HCC. (A) Targets of miR-29c-3p were predicted using the miRWalk, starBase, Oncomir, TargetScan and miRDB databases. (B) Expression heatmap of 16 target genes of miR-29c-3p was analyzed in HCC using the UALCAN database. (C) Binding sites between miR-29c-3p and NASP. (D) Relationship between miR-29c-3p and NASP was confirmed by dual luciferase reporter assay. (E) NASP expression was detected by reverse transcription-quantitative PCR in HCC and normal tissues. NASP expression level analysis in (F) GEPIA and (G) UALCAN databases, respectively. (H) HCC tumor grade was positively associated with NASP expression. (I) Expression of NASP at different HCC patient stages. (J) NASP expression was related to the disease-free survival of patients with HCC in GEPIA database. ${ }^{* *} \mathrm{P}<0.01$ and ${ }^{* * *} \mathrm{P}<0.001$ vs. WT or Normal. miR, microRNA; NASP, nuclear autoantigenic sperm protein; HCC, hepatocellular carcinoma; WT, wild-type; MUT, mutant-type; GEPIA, Gene Expression Profiling Interactive Analysis.

the overexpression of miR-29c-3p markedly reduced the luciferase activity of cells transfected with CRNDE-WT, but not CRNDE-MUT (Fig. 3D). Additionally, miR-29c-3p expression was markedly decreased in HCC tissues (Fig. 3E). To determine the effects of miR-29c-3p on CRNDE expression, miR-29c-3p expression was assessed in HCC cells transfected with miR-29c-3p mimics or inhibitor. The results showed that miR-29c-3p mimics significantly increased miR-29c-3p expression, while the miR-29c-3p inhibitor downregulated expression (Fig. S1A and B). Furthermore, CRNDE expression was inhibited by miR-29c-3p overexpression in HCC cells (Fig. 3F). Reduced expression of miR-29c-3p was also observed in patients with $\mathrm{HCC}$ via bioinformatics analysis (Fig. 3G). Collectively, these results confirm that miR-29c-3p is a direct target of CRNDE, and is decreased in HCC.

NASP is a downstream target gene of miR-29c-3p. The downstream target genes of miR-29c-3p were predicted using five databases, and a total of 17 genes co-existed in five databases (Fig. 4A). The heat map of gene expression indicated that the expression levels of NASP, TMOD3, KCTD2 and CPR37 were higher in HCC compared with the other genes investigated (Fig. 4B). In the present study, a binding site between miR-29c-3p and NASP was predicted using TargetScan (Fig. 4C). Furthermore, dual-luciferase reporter assays confirmed that the luciferase activity of NASP-WT-transfected cells was significantly decreased following the overexpression of miR-29c-3p, while miR-29c-3p mimics did not affect the luciferase activity of the NASP-MUT group (Fig. 4D). RT-qPCR analysis indicated that NASP mRNA was significantly increased in HCC compared with normal tissues (Fig. 4E). Similarly, higher NASP expression levels were also observed in HCC cancer tissues via the GEPIA and UALCAN databases, respectively (Fig. 4F and G). Moreover, NASP expression was positively associated with HCC tumor grade and individual 
A

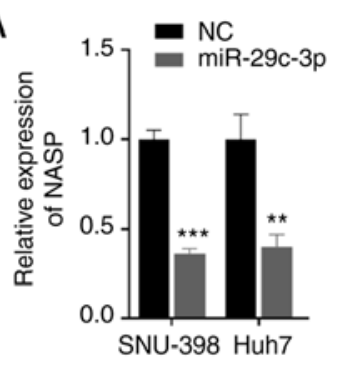

B

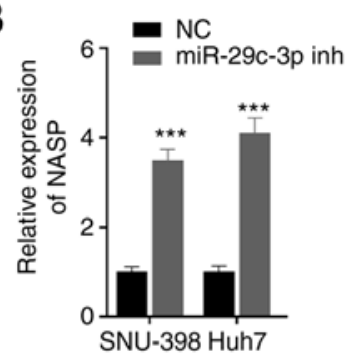

C

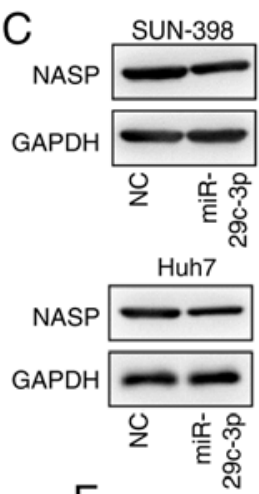

E
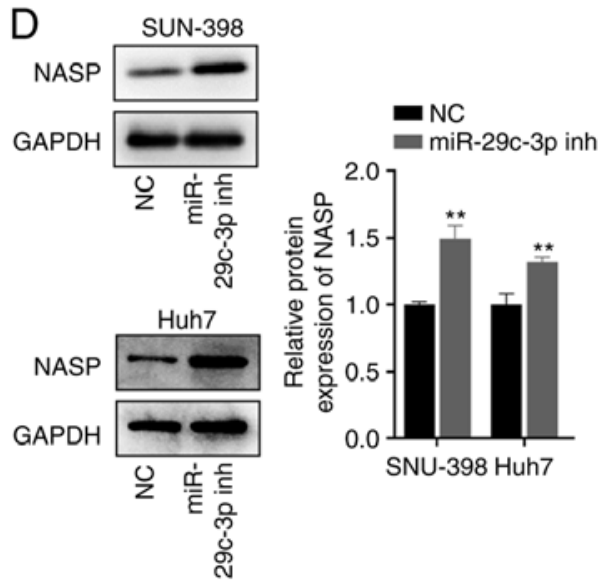

\section{- shNC}

- ShCRNDE

- shCRNDE+miR-29c-3p inh

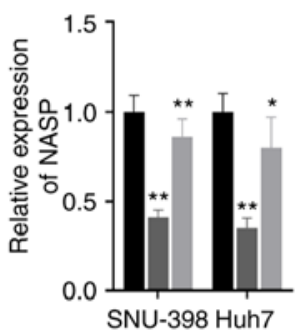

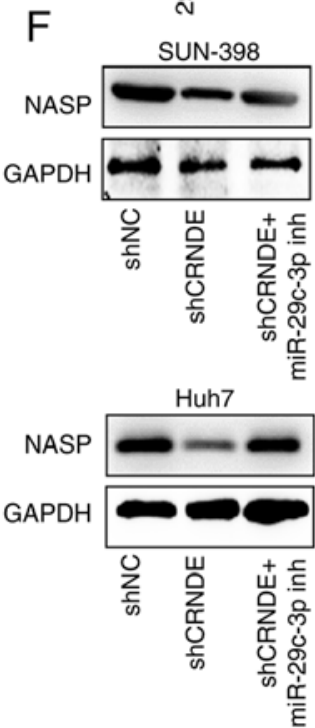

I

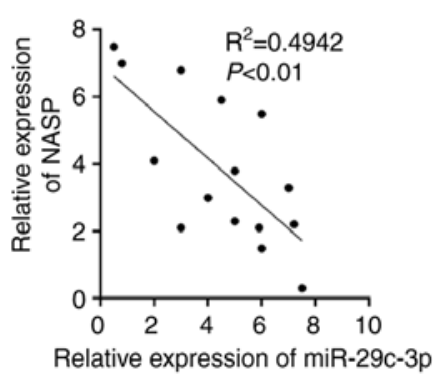

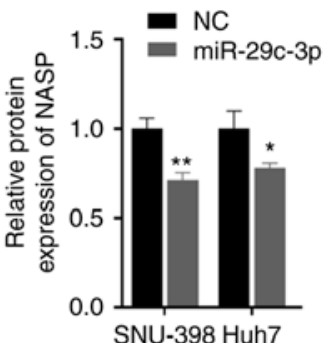

- shNC

- shCRNDE

- shCRNDE+miR-29c-3p inh

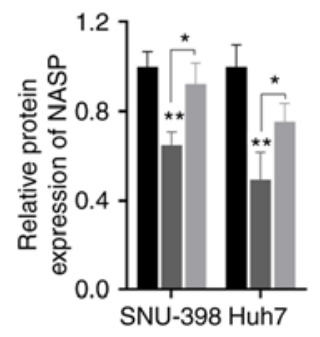

G

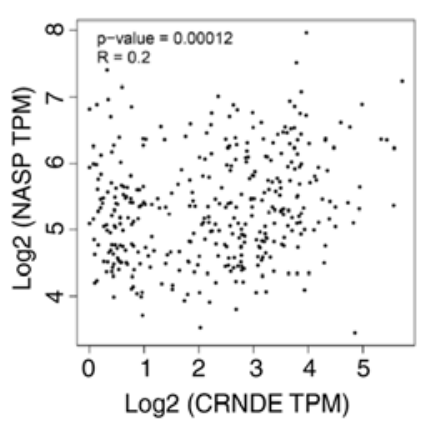

$\mathrm{H}$

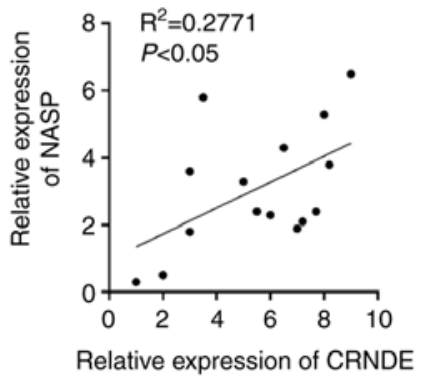

Figure 5. CRNDE and miR-29c-3p regulate NASP expression in HCC cells. (A and B) Reverse transcription-quantitative PCR was used to analyze NASP expression in miR-29c-3p mimics/inhibitor-transfected HCC cells. (C and D) Protein expression of NASP was analyzed by western blotting in miR-29c-3p mimics/inhibitor transfected HCC cells. Effect of CRNDE and miR-29c-3p on NASP expression at the (E) mRNA and (F) protein levels in cells transfected with shNC, shCRNDE or shCRNDE+miR-29c-3p inhibitor. (G and H) Correlation analysis between NASP and CRNDE in HCC tissues. (I) Negative correlation between NASP and miR-29c-3p expression in HCC. (J) Immunohistochemistry was used to detect the expression of NASP in the tumor tissues of patients with HCC. ${ }^{*} \mathrm{P}<0.05,{ }^{* *} \mathrm{P}<0.01$ and ${ }^{* * *} \mathrm{P}<0.001$ vs. shNC, unless otherwise indicated. miR, microRNA; CRNDE, lncRNA colorectal neoplasia differentially expressed; NASP, nuclear autoantigenic sperm protein; HCC, hepatocellular carcinoma; sh, short hairpin (RNA); inh, inhibitor.

cancer stage (Fig. 4H and I), and HCC patients with higher NASP expression experienced lower disease-free survival rates (Fig. 4J).

$C R N D E$ and $m i R-29 c-3 p$ regulate NASP expression in HCC cells. To determine the effects of CRNDE and miR-29c-3p on NASP expression, HCC cells were transfected with miR-29c-3p mimics, miR-29c-3p inhibitor, shCRNDE, CRNDE or their respective negative controls. The results showed that NASP expression was inhibited by miR-29c-3p mimics (Fig. 5A), but increased by the miR-29c-3p inhibitor (Fig. 5B). Consistent with these results, the protein expression levels of NASP were regulated by miR-29c-3p mimics and inhibitor (Fig. 5C and D). Furthermore, the effect of CRNDE on NASP expression was assessed in HCC cells transfected with shCRNDE or CRNDE. The results indicated that NASP expression at the mRNA and protein levels were notably suppressed by shCRNDE, which was rescued by the miR-29c-3p inhibitor (Fig. 5E and F). Additionally, correlation analyses revealed that NASP expression was positively associated with that of CRNDE, (Fig. 5G and H), but negatively associated with miR-29c-3p expression (Fig. 5I). Immunohistochemistry also revealed that the expression of NASP was also increased in the tumor tissues of patients with HCC, compared with normal tissues 

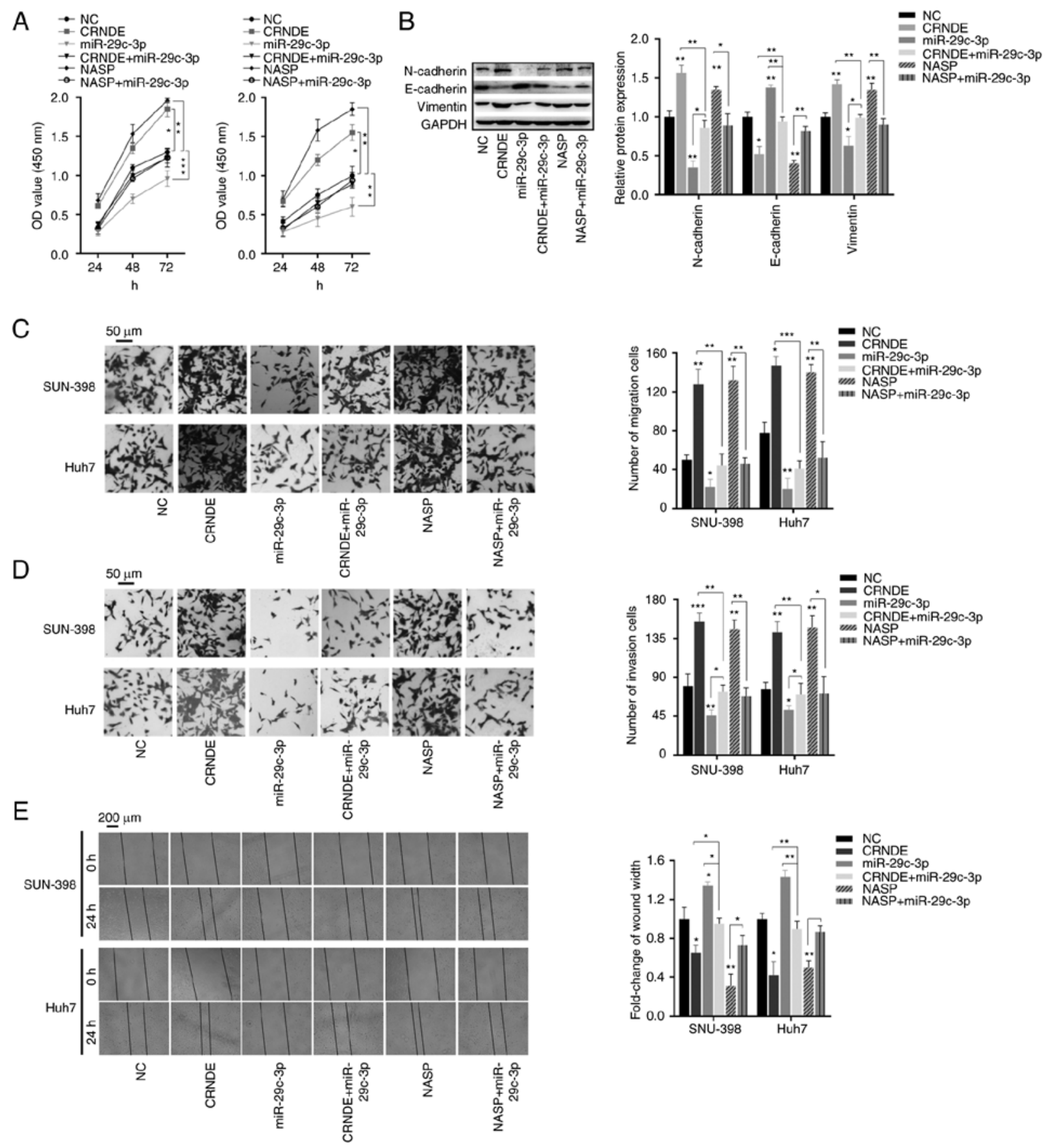

Figure 6. CRNDE-miR-29c-3p-NASP axis regulates HCC cell proliferation, migration and invasiveness. HCC cells were transfected with CRNDE, miR-29c-3p mimics, NASP, CRNDE+miR-29c-3p mimics, NASP+miR-29c-3p mimics or the corresponding negative controls. (A) Proliferation ability of HCC cells following transfection. (B) Expression levels of epithelial-mesenchymal transition-related proteins were detected by western blotting in HCC cells following transfection. Transwell assays were performed to evaluate the (C) migration and (D) invasiveness of HCC cells following transfection. (E) Wound-healing assays were used to analyze HCC cell migration. ${ }^{*} \mathrm{P}<0.05,{ }^{* *} \mathrm{P}<0.01$ and ${ }^{* * *} \mathrm{P}<0.001$. miR, microRNA; CRNDE, lncRNA colorectal neoplasia differentially expressed; NASP, nuclear autoantigenic sperm protein; HCC, hepatocellular carcinoma; sh, short hairpin (RNA).

(Fig. 5J). Thus, these results indicated that CRNDE regulated NASP expression by inhibiting miR-29c-3p in HCC cells.

The CRNDE-miR-29c-3p-NASP axis regulates HCC cell proliferation, migration and invasiveness. To further determine the roles of the CRNDE-miR-29c-3p-NASP axis in $\mathrm{HCC}$, the overexpression efficiency of CRNDE and NASP was detected in HCC cells (Fig. S1C and D). Then, the indicated transfections were performed, and rescue assays were conducted to study the effects of miR-29c-3p on CRNDE and NASP. Firstly, CCK- 8 assays indicated that cellular proliferation ability was promoted in SNU-398 and Huh7 cells following CRNDE and NASP overexpression, while the opposite effect was observed following miR-29c-3p mimics transfection. However, rescue assays indicated that the promotion of cell proliferation caused by CRNDE and NASP was significantly weakened by miR-29c-3p mimics (Fig. 6A). To evaluate whether the CRNDE-miR-29c-3p-NASP axis affects epithelial mesenchymal transition (EMT), western blot analysis was carried out. The results indicated that the 
expression levels of $\mathrm{N}$-cadherin and vimentin were increased in cells transfected with the CRNDE or NASP overexpression plasmids, while E-cadherin was downregulated. However, the effects of CRNDE or NASP in EMT was reversed by miR-29c-3p mimics. These results demonstrated that the overexpression of CRNDE and NASP was conducive to EMT, but was negatively regulated by miR-29c-3p mimics (Fig. 6B). Moreover, migration and wound-healing assays indicated that CRNDE and NASP promoted cellular migration capacity, which was reversed by miR-29c-3p mimics (Fig. 6C and E). Consistent with the migration results, the overexpression of miR-29c-3p also successfully rescued the effects of CRNDE and NASP on cellular invasiveness (Fig. 6D). Collectively, these data demonstrated that CRNDE promoted HCC cell proliferation, migration and invasiveness by regulating the miR-29c-3p/NASP axis.

\section{Discussion}

LncRNAs serve critical roles in various diseases, including cancer. As such, dysregulation of lncRNAs is closely involved in the tumorigenesis and clinical prognosis of cancer (25). The IncRNA-mediated progression of HCC has been widely studied. For example, knockdown of lncRNA HOTAIR suppressed glycolysis by targeting the miR-130a-3p/HIF1A axis in HCC during hypoxia (26). As a metastasis-associated molecule, IncRNA AY927503 was found to be highly expressed and to correlate with tumor metastasis and poor prognosis in patients with HCC by stimulating ITGAV transcription (27). Furthermore, IncRNA SNHG16 accelerated HCC progression by regulating the expression of autophagy-related gene P62 via miR-17-5p (28). In addition, lncRNA 1ncTCF7 activated wnt signaling to promote the self-renewal of liver cancer stem cells (29). In the present study, CRNDE was found to be significantly upregulated in HCC compared with adjacent-normal tissues, suggesting that CRNDE is an HCC-related lncRNA. These findings were consistent with previous studies demonstrating that CRNDE functions as an oncogene in multiple cancers, such as pancreatic cancer (30), hepatocellular carcinoma (31) and glioma (32). Thus, the function and clinical significance of CRNDE warrants further investigation.

In loss-of-function assays, CRNDE-knockdown was found to markedly suppress the proliferation, migration and invasiveness of $\mathrm{HCC}$ cells, illustrating the oncogenic role of CRNDE in HCC. However, the study did not reveal the role of CRNDE in HCC in vivo, which requires additional investigation. Furthermore, the molecular mechanism underlying the effects of CRNDE in HCC remains unclear.

It is well known that lncRNAs perform their functions via various mechanisms, such as RNA-protein interaction, RNA-RNA pathways and the ceRNA pathway. In the present study, miR-29c-3p was predicted as a target of CRNDE in HCC. As such, a luciferase reporter assay confirmed a direct interaction between CRNDE and miR-29c-3p. In previous studies, miR-29c-3p was competitively bound by lncRNA RP11-480I12.5 to inhibit cancer progression (33). As a tumor suppressor, miR-29c-3p was negatively regulated by lncRNA MYOSLID in gastric cancer (34). Combining the revelation that miR-29c-3p expression was decreased in HCC cells in the present study, miR-29c-3p was confirmed as a tumor suppressor in HCC. Thus, we hypothesize that CRNDE plays an oncogenic role in $\mathrm{HCC}$ by inhibiting miR-29c-3p expression.

Previous studies have indicated that the overexpression of NASP promotes the proliferation, migration and invasiveness of cancer cells (35). The carcinogenesis of NASP was also discovered in gastric cancer (19), ovarian cancer (20) and HCC (21). In the present study, NASP was identified as a direct target of miR-29c-3p in HCC cells. NASP was also highly expressed in HCC tissues, which indicated poor disease-free survival rates of patients with HCC. These findings demonstrated that NASP plays the role of tumor promoter in HCC. Furthermore, miR-29c-3p negatively regulated NASP expression in HCC cells, but CRNDE exerted a positive effect on NASP expression. Moreover, the positive and negative correlation of CRNDE or miR-29c-3p on NASP, respectively, were determined in HCC tissues. The overexpression of miR-29c-3p inhibited CRNDE and NASP expression, and reduced the enhancement of CRNDE- or NASP-associated proliferation,EMT, migration and invasiveness of HCC cells. Thus, the results of the present study highlighted the effect of the CRNDE/miR-29c-3p/NASP axis in HCC. The potential role of the CRNDE/miR-29c-3p/NASP axis in $\mathrm{HCC}$ in vivo will be investigated in future studies.

In summary, the results of the present study indicated that CRNDE promoted the progression of HCC by inhibiting cellular proliferation, migration and invasiveness. This was achieved via direct targeting of miR-29c-3p to upregulate NASP expression. Thus, the CRNDE/miR-29c-3p/NASP axis may be a potential therapeutic target for HCC.

\section{Acknowledgements}

Not applicable.

\section{Funding}

No funding was received.

\section{Availability of data and materials}

The datasets used and/or analyzed during the current study are available from the corresponding author on reasonable request.

\section{Authors' contributions}

GXi, YZ and BL designed and conceived the study. YZ, BL, $\mathrm{GXu}$ and $\mathrm{CH}$ conducted the experiments. All authors analyzed the data. GXi, YZ and BL drafted the manuscript. All authors have read and approved the final manuscript. GXi and YZ confirmed the authenticity of all the raw data.

\section{Ethics approval and consent to participate}

The present study was approved by the Ethical Committee of the Second Hospital of Tianjin Medical University. Written informed consent was obtained from all patients.

\section{Patient consent for publication}

Not applicable. 


\section{Competing interests}

The authors declare that they have no competing interests.

\section{References}

1. Bray F, Ferlay J, Soerjomataram I, Siegel RL, Torre LA and Jemal A: Global cancer statistics 2018: GLOBOCAN estimates of incidence and mortality worldwide for 36 cancers in 185 countries. CA Cancer J Clin 68: 394-424, 2018.

2. Yu LX and Schwabe RF: The gut microbiome and liver cancer: Mechanisms and clinical translation. Nat Rev Gastroenterol Hepatol 14: 527-539, 2017.

3. Lau WY and Lai EC: Hepatocellular carcinoma: Current management and recent advances. Hepatobiliary Pancreat Dis Int 7: 237-257, 2008

4. Portolani N, Coniglio A, Ghidoni S, Giovanelli M, Benetti A, Tiberio GA and Giulini SM: Early and late recurrence after liver resection for hepatocellular carcinoma: Prognostic and therapeutic implications. Ann Surg 243: 229-235, 2006.

5. Kulik L and El-Serag HB: Epidemiology and management of hepatocellular carcinoma. Gastroenterology 156: 477-491.e1, 2019

6. Mercer TR, Dinger ME and Mattick JS: Long non-coding RNAs: Insights into functions. Nat Rev Genet 10: 155-159, 2009.

7. Zeng Z, Dong J, Li Y, Dong Z, Liu Z, Huang J, Wang Y, Zhen Y and $\mathrm{Lu} Y$ Y: The expression level and clinical significance of IncRNA X91348 in hepatocellular carcinoma. Artif Cells Nanomed Biotechnol 47: 3067-3071, 2019.

8. Yang MH, Zhao L, Wang L, Ou-Yang W, Hu SS, Li WL, Ai ML, Wang YQ, Han Y, Li TT, et al: Nuclear lncRNA HOXD-AS1 suppresses colorectal carcinoma growth and metastasis via inhibiting HOXD3-induced integrin $\beta 3$ transcriptional activating and MAPK/AKT signalling. Mol Cancer 18: 31, 2019.

9. Mu M, Niu W, Zhang X, Hu S and Niu C: LncRNA BCYRN1 inhibits glioma tumorigenesis by competitively binding with miR-619-5p to regulate CUEDC2 expression and the PTEN/AKT/p21 pathway. Oncogene 39: 6879-6892, 2020.

10. Li H, Jia J, Yang L, Chu J, Sheng J, Wang C, Meng W, Jia Z, Yin H, Wan J and He F: LncRNA MIR205HG drives esophageal squamous cell carcinoma progression by regulating miR-214/SOX4 axis. Onco Targets Ther 13: 13097-13109, 2020.

11. Liu Z, Zhou W, Lin C, Wang X, Zhang X, Zhang Y, Yang R, Chen W and Cao W: Dysregulation of FOXD2-AS1 promotes cell proliferation and migration and predicts poor prognosis in oral squamous cell carcinoma: A study based on TCGA data. Aging (Albany NY) 13: 2379-2396, 2020.

12. Kan Z, Zheng H, Liu X, Li S, Barber TD, Gong Z, Gao H, Hao K, Willard MD, Xu J, et al: Whole-genome sequencing identifies recurrent mutations in hepatocellular carcinoma. Genome Res 23: 1422-1433, 2013.

13. Wang Y, Lu JH, Wu QN, Jin Y, Wang DS, Chen YX, Liu J, Luo XJ, Meng Q, Pu HY, et al: LncRNA LINRIS stabilizes IGF2BP2 and promotes the aerobic glycolysis in colorectal cancer. Mol Cancer 18: 174, 2019.

14. Li Z, Hou P, Fan D, Dong M, Ma M, Li H, Yao R, Li Y, Wang G, Geng P, et al: The degradation of EZH2 mediated by lncRNA ANCR attenuated the invasion and metastasis of breast cancer. Cell Death Differ 24: 59-71, 2017.

15. Han P, Li JW, Zhang BM, Lv JC, Li YM, Gu XY, Yu ZW, Jia YH, Bai XF, Li L, et al: The lncRNA CRNDE promotes colorectal cancer cell proliferation and chemoresistance via miR-181a-5p-mediated regulation of $\mathrm{Wnt} / \beta$-catenin signaling. Mol Cancer 16: 9, 2017.

16. Zhang F, Wang H, Yu J, Yao X, Yang S, Li W, Xu L and Zhao L: LncRNA CRNDE attenuates chemoresistance in gastric cance via SRSF6-regulated alternative splicing of PICALM. Mol Cancer 20: 6, 2021.

17. Chen C, Huang Z, Mo X, Song Y, Li X, Li X and Zhang M: The circular RNA 001971/miR-29c-3p axis modulates colorectal cancer growth, metastasis, and angiogenesis through VEGFA. J Exp Clin Cancer Res 39: 91, 2020.
18. Wu H, Zhang W, Wu Z, Liu Y, Shi Y, Gong J, Shen W and Liu C: miR-29c-3p regulates DNMT3B and LATS1 methylation to inhibit tumor progression in hepatocellular carcinoma. Cell Death Dis 10: 48, 2019.

19. Yu B, Chen X, Li J, Gu Q, Zhu Z, Li C, Su L and Liu B microRNA-29c inhibits cell proliferation by targeting NASP in human gastric cancer. BMC Cancer 17: 109, 2017.

20. Ali-Fehmi R, Chatterjee M, Ionan A, Levin NK, Arabi H, Bandyopadhyay S, Shah JP, Bryant CS, Hewitt SM, O'Rand MG, et al: Analysis of the expression of human tumor antigens in ovarian cancer tissues. Cancer Biomark 6: 33-48, 2010.

21. Kang X, Feng Y, Gan Z, Zeng S, Guo X, Chen X, Zhang Y, Wang $\mathrm{C}$, Liu K, Chen X, et al: NASP antagonize chromatin accessibility through maintaining histone $\mathrm{H} 3 \mathrm{~K} 9$ mel in hepatocellular carcinoma. Biochim Biophys Acta Mol Basis Dis 1864: 3438-3448, 2018.

22. Livak KJ and Schmittgen TD: Analysis of relative gene expression data using real-time quantitative PCR and the 2(-Delta Delta C(T)) method. Methods 25: 402-408, 2001

23. Wang L, Cho KB, Li Y, Tao G, Xie Z and Guo B: Long noncoding RNA (lncRNA)-mediated competing endogenous RNA networks provide novel potential biomarkers and therapeutic targets for colorectal cancer. Int J Mol Sci 20: 5758, 2019.

24. Chan JJ and Tay Y: Noncoding RNA:RNA regulatory networks in cancer. Int J Mol Sci 19: 1310, 2018.

25. He Y, Meng XM, Huang C, Wu BM, Zhang L, Lv XW and Li J: Long noncoding RNAs: Novel insights into hepatocelluar carcinoma. Cancer Lett 344: 20-27, 2014.

26. Hu M, Fu Q, Jing C, Zhang X, Qin T and Pan Y: LncRNA HOTAIR knockdown inhibits glycolysis by regulating miR-130a-3p/HIF1A in hepatocellular carcinoma under hypoxia. Biomed Pharmacother 125: 109703, 2020.

27. Kang CL, Qi B, Cai QQ, Fu LS, Yang Y, Tang C, Zhu P, Chen QW, Pan J, Chen MH and Wu XZ: LncRNA AY promotes hepatocellular carcinoma metastasis by stimulating ITGAV transcription. Theranostics 9: 4421-4436, 2019.

28. Zhong JH, Xiang X, Wang YY, Liu X, Qi LN, Luo CP, Wei WE, You XM, Ma L, Xiang BD and Li LQ: The IncRNA SNHG16 affects prognosis in hepatocellular carcinoma by regulating p62 expression. J Cell Physiol 235: 1090-1102, 2020.

29. Wang Y, He L, Du Y, Zhu P, Huang G, Luo J, Yan X, Ye B, Li C, Xia P, et al: The long noncoding RNA lncTCF7 promotes self-renewal of human liver cancer stem cells through activation of Wnt signaling. Cell Stem Cell 16: 413-425, 2015.

30. Wang G, Pan J, Zhang L, Wei Y and Wang C: Long non-coding RNA CRNDE sponges miR-384 to promote proliferation and metastasis of pancreatic cancer cells through upregulating IRS1. Cell Prolif 50: e12389, 2017.

31. Ji D, Jiang C, Zhang L, Liang N, Jiang T, Yang B and Liang H: LncRNA CRNDE promotes hepatocellular carcinoma cell proliferation, invasion, and migration through regulating miR-203/ BCAT1 axis. J Cell Physiol 234: 6548-6560, 2019.

32. Zheng J, Liu X, Wang P, Xue Y, Ma J, Qu C and Liu Y: CRNDE promotes malignant progression of Glioma by attenuating miR-384/PIWIL4/STAT3 axis. Mol Ther 24: 1199-1215, 2016.

33. Lou W, Ding B, Zhong G, Yao J, Fan W and Fu P: RP11-480I12.5-004 promotes growth and tumorigenesis of breast cancer by relieving miR-29c-3p-mediated AKT3 and CDK6 degradation. Mol Ther Nucleic Acids 21: 916-931, 2020.

34. Han Y, Wu N, Jiang M, Chu Y, Wang Z, Liu H, Cao J, Liu H, $\mathrm{Xu} \mathrm{B}$ and Xie X: Long non-coding RNA MYOSLID functions as a competing endogenous RNA to regulate MCL-1 expression by sponging miR-29c-3p in gastric cancer. Cell Prolif 52: el2678, 2019.

35. Kong F, Li L, Wang C, Zhang Q and He S: MiR-381-3p suppresses biological characteristics of cancer in head-neck squamous cell carcinoma cells by targeting nuclear autoantigenic sperm protein (NASP). Biosci Biotechnol Biochem 84: 703-713, 2020.

This work is licensed under a Creative Commons Attribution-NonCommercial-NoDerivatives 4.0 International (CC BY-NC-ND 4.0) License. 